\title{
Resistance to Adoption of Best Practices
}

\section{A Report from the Berkeley Institute for Data Science's Best Practices in Data Science Series}

${ }^{*}$ Corresponding author: dsholler@berkeley.edu

${ }^{\dagger}$ These authors contributed equally to this work

₹These authors contributed equally to this work, order is alphabetical

Published: 12 Mar 2018

DOI: $10.31235 /$ osf.io/qr8cz

License: Creative Commons Attribution (CC BY 4.0 Intl)
Dan Sholler ${ }^{1,2{ }^{\star}}$, Sara Stoudt ${ }^{1,3 \dagger}$, Chris Kennedy ${ }^{1,4,5 \neq}$, Fernando Hoces de la Guardia ${ }^{6 \ddagger}$, François Lanusse ${ }^{1,7,8 \ddagger}$, Karthik Ram ${ }^{1,2,9 \ddagger}$, Kellie Ottoboni ${ }^{1,3 \ddagger}$, Marla Stuart ${ }^{1,10 \ddagger}$, Maryam Vareth ${ }^{1,11 \ddagger}$, Nelle Varoquaux ${ }^{1,3 \ddagger}$, Rebecca Barter $^{1,3 \ddagger}$, R. Stuart Geiger ${ }^{1 \ddagger}$, Scott Peterson ${ }^{12 \ddagger}$, Stéfan van der Walt ${ }^{1 \ddagger}$

${ }^{1}$ Berkeley Institute for Data Science, University of California, Berkeley

2 rOpenSci

3 Department of Statistics, University of California, Berkeley

${ }^{4}$ D-Lab, University of California, Berkeley

${ }^{5}$ Division of Biostatistics, University of California, Berkeley

${ }^{6}$ Berkeley Initiative for Transparency in the Social Sciences, University of California, Berkeley

7 Berkeley Center for Cosmological Physics, University of California, Berkeley

8 Foundations of Data Analysis Institute, University of California, Berkeley

9 Berkeley Initiative in Global Change Biology, University of California, Berkeley

10 School of Social Welfare, University of California, Berkeley

11 Department of Radiology and Biomedical Imaging, University of California, San Francisco

12 Morrison Library and Graduate Services Library, University of California, Berkeley

Abstract:

There are many recommendations of "best practices" for those doing data science, data-intensive research, and research in general. These documents usually present a particular vision of how people should work with data and computing, recommending specific tools, activities, mechanisms, and sensibilities. However, implementation of best (or better) practices in any setting is often met with resistance from individuals and groups, who perceive some drawbacks to the proposed changes to everyday practice. We offer some definitions of resistance, identify the sources of researchers' hesitancy to adopt new ways of working, and describe some of the ways resistance is manifested in data science teams. We then offer strategies for overcoming resistance based on our group members' experiences working alongside resistors or resisting change themselves. Our discussion concluded with many remaining questions left to tackle, some of which are listed at the end of this piece.

Recommended citation: Dan Sholler, Sara Stoudt, Chris Kennedy, Fernando Hoces de la Guardia, François Lanusse, Karthik Ram, Kellie Ottoboni, Marla Stuart, Maryam Vareth, Nelle Varoquaux, Rebecca Barter, R. Stuart Geiger, Scott Peterson, and Stéfan van der Walt. "Resistance to Adoption of Best Practices." BIDS Best Practices in Data Science Series. Berkeley Institute for Data Science: Berkeley, California. 2019. 10.31235/osf.io/qr8cz 


\section{Introduction}

In prior Best Practices in Data Science meetings, we have discussed some of the challenges of doing data-intensive research in teams (Geiger et al., 2018b), including managing turnover (Sholler et al., 2019); fostering a diverse and inclusive data science team (Geiger et al., 2018a), and creating and sustaining robust workflows. We have steadily developed some best (or better) practices for dealing with these challenges, as have other researchers and practitioners in academia and industry (Wilson et al., 2014; Goodman et al., 2014; Sandve et al., 2013).

However, implementation of best (or even "good enough" (Wilson et al., 2017)) practices in any setting is often met with resistance from individuals and groups, who perceive some drawbacks to the proposed changes to everyday practice. We offer some definitions of resistance, identify the sources of researchers' hesitancy to adopt new ways of working, and describe some of the ways resistance is manifested in data science teams. We then offer strategies for overcoming resistance based on our group members' experiences working alongside resistors or resisting change themselves. Our discussion concluded with many remaining questions left to tackle, some of which are listed at the end of this piece.

\section{What is resistance?}

Resistance is a common phenomenon that arises in response to change. While we might "know it when we see it," resistance is also a somewhat-thoroughly studied theoretical concept developed in the information systems and organization science literatures. Research interest in resistance is driven by its costly impact on organizational change efforts, particularly as it relates to the implementation of a new enterprise technology (e.g. Joshi, 1991; Lapointe and Rivard, 2005; Marakas and Hornik, 1996; Markus, 1983). In general, this literature ascribes acts of resistance to perceived loss of power, unwanted changes to everyday practice, and overall stress about the possibility of change. The perceived threat of a new technology or practice can lead to active avoidance or misuse, ultimately jeopardizing the goal of the change effort. As we describe below, each of these elements is visible in efforts to implement best practices in data-intensive research teams.

\section{Sources of resistance to best practices}

Building on what constitutes resistance, we turned our attention to why members of data-intensive research teams might resist new modes of working with data. Some of the reasons, such as inertia, are intuitive: Researchers don't want to change 
long-held practices that have presumably worked for carrying out their work in the past. Other reasons, such as concerns about the longevity of a new practice or tool, are rooted in skepticism about "fads" in science, generally, and scientific computing, specifically.

\section{Inertia, or "I've been doing it this way my entire career"}

Perhaps the most obvious reason for resistance to adoption of best practices is inertia, or the tendency for core research practices to endure over time. Several group members noted that efforts to engage collaborators with new approaches to data management, data manipulation and analysis, manuscript writing, and other research tasks were met with resistance, often in the form of collaborators sticking to their existing practices. In some cases, resistors justified their hesitancy because the proposed new practice or tool didn't match the full capabilities of the original (e.g., track changes is often a sticking point in attempts to move collaborative writing toward LaTeX or Google Drive). Even though many aspects of the workflow may be made easier, more reproducible, and/or more transparent by the new tool, a bottleneck remains. This deters users from adoption to avoid switching back and forth between "best" and functional.

\section{Time and resource constraints}

Even if team members welcome the idea of change, the switching costs might be perceived as too high to justify adopting new practices. For example, consider a scenario in which a new programming environment or software package offers enhanced capabilities to analyze data. Who is responsible for the time and cost of converting the team's previous work to appropriate formats? Labs with decades of work could conceivably need to create a staff or postdoc position focused solely on conversion, an effort that could detract from research efforts and be difficult to justify to some funders.

Relatedly, we discussed a theme that has persisted throughout all of our Best Practices sessions: adoption of best practices breaks down in time crunches. In other words, finding the "right" time to adopt new practices is situationally difficult, as research teams are continually and simultaneously writing grants, collecting data, conducting analyses, writing up results, and otherwise balancing time-sensitive tasks. This juggling act leaves little time for learning new practices, particularly for practices with steep learning curves. And when deadlines loom, researchers seem to be more likely to revert to old, comfortable practices to meet demands. 


\section{Worry about longevity of new approaches}

Researchers might also have concerns that a new practice or tool is merely a passing fad rather than a paradigmatic shift in the way research is done. Several group members raised this point as an example of why they or their collaborators resist change. The issue of the longevity of a given best practice is often implicit in how people talk about changes in data-intensive science, yet there are few resources for evaluating the long-term potential of tools and approaches. Researchers might feel more comfortable working with software backed by paid technical support, for example, than working with software supported by a distributed open source community.

\section{Forms of resistance}

No matter the source of resistance, researchers tend to resist in similar ways. Resistance, both in the literature on organizational change and in our group members' experiences, tends to take the form of outright refusal (either individually or by multiple members of the team), of vocalized disdain or hesitancy, or of subtly sabotaging efforts to shift practices by bottlenecking the change process.

\section{Avoidance and refusal}

A common form of resistance is simple avoidance of the tool or practice or a refusal to update a skillset. A poignant, frequently discussed example is some researchers' reluctance to switch from proprietary software to open source software (e.g. Stata, SAS, SPSS $\rightarrow$ R, Matlab $\rightarrow$ Python, Microsoft Office $\rightarrow$ LaTeX, Google Suite). We often frame examples like these as a problem of technical aptitude. Not every researcher pays mind to coding or workflow issues; rather, they view these practices as means to an end, and motivation and flexibility to change tools may be lacking.

\section{Bottlenecking}

Perhaps as a result of avoidance, team projects are limited by their most resistant member. In a collaborative project, it can be difficult to coordinate work if members are using different tools (e.g. different team members writing up results in Word, Google Docs, and LaTeX). This is especially true for interdisciplinary teams where members bring different norms from their respective fields. In these situations, one or more people often has to serve as the go-between and manage the overhead work of converting between formats or ensuring compatibility between workflows. In severe cases, resistors can bottleneck the work by using a practice or tool that does not align with what the rest of the team uses. Then, the path of least resistance 
often becomes agreeing on a tool or practice that is not "best" as defined by the majority of the team.

\section{Vocalized Resistance}

Collaborators may go beyond bottlenecking a project or engaging in other passive forms resistance. Another form of resistance in team and community settings occurs when a member actively lobbies against the adoption of or complains about a particular tool or practice. Any energy or momentum for change is often used up in debating rather than moving towards a new practice. This form of resistance can be especially tricky to navigate given disparate power dynamics of the vocal members of the community.

\section{Strategies for overcoming resistance}

\section{Demonstrating value}

Evidence that the best practice will help solve a problem, save time, and/or enhance reproducibility and transparency can alleviate resistance. Demonstrating problemdriven value in both the short- and long-term is key. For example, if there is a particular recurring pain point that can be overcome with a switch to a new tool, a quick change may be perceived as justified by members of the team. Finding these pain points and addressing them with the new practice-even if the problem is not the primary target of the change-can help to sway resistors. These "quick hits" can supplement efforts to show that a change will lead to increased productivity or efficiency in the long run and promote openness to trying out the new practice.

A related approach to demonstrating value is showcasing a polished product, such as a beautiful new visualization of old data or a speedy re-analysis. These attentiongrabbing demonstrations can entice a resistant person to consider a new tool or practice. Following the compelling, potentially more complicated, example with a set of distilled, smaller and easier examples will familiarize the user with the new tool and illustrate its accessibility.

\section{Offering credit and rewards}

If the benefits of switching to the best practice are hidden or not equitably distributed among team members, the time spent learning a new tool can seem like a waste without an obvious or immediate payoff. Treating the best practice as part of professional development and having dedicated time set aside to learn new skills 
can transform the act of learning from a burden to a reward. The Carpentries ${ }^{1}$ organization provides a high-profile example of how to make computational skill-building a rewarding experience. Another, related way to "get credit" for updating one's skillset is to create some output throughout the learning process. This approach can include something informal (e.g. a series of blog posts documenting the process and establishing a record of an evolving skill set) or formal (e.g. a handbook or methods paper introducing the new tool or workflow to other researchers).

\section{Mandates}

The above strategies can be employed within small or large research teams. Moving entire disciplines toward best practices, though, likely requires some action from powerful institutions in the field. Our group discussed the potential impact of journal policies for manuscripts (e.g., requiring submissions to be in LaTeX or archiving sufficient data and code) on shifting researchers toward best practices. Funders have also begun to take steps toward requiring researchers to engage in what funding agencies view as best practices, such as mandating data management plans in all proposals or urging teams to house their data in appropriate repositories. To be sure, principal investigators may hold some similar mandate power within their own labs (as we have mentioned in previous series posts).

\section{Procedural justice and providing support}

Studies in organizational behavior have demonstrated that people are more satisfied with the outcome of a decision when they perceive justice in the decision-making process, no matter the positive or negative valence of the decision's outcome (e.g. Folger and Konovsky, 1989; McFarlin and Sweeney, 1992). In this vein, researchers might be less resistant to changes to their practice if they have input in how the new practice is selected and implemented. Involving the entire research team in decisions like software selection and workflow design, then, provides a way of minimizing friction when a change is made. Providing support for researchers post-adoption is equally important. Having access to regular help and timely troubleshooting as a user faces the learning curve of a new tool or practice can help a resistant person become more confident in making a change to their workflow. Whether assistance comes from paid services or members of an open source community, a guaranteed support system can make a difference in the willingness to move towards best practices.

\footnotetext{
${ }^{1}$ https://thecarpentries.org
} 


\section{Summary and Conclusions}

Data-intensive research teams should expect to encounter some resistance when changing practices. Comfort and familiarity with existing ways of working, hesitancy to invest time and resources into switching, and concern over the long-term viability of the new practice all factor into researcher decisions to resist. Faced with resistance, teams can employ some of the above strategies to push along changes and improvements to practice. We have covered some of the approaches that might be used, but we are sure there are many more and welcome any additions and feedback on what we have presented here. In particular, we hope to spark discussion on some remaining questions:

- What is a best practice, anyway? We spent some time considering this question. We, as a group, tend to equate the use of open source tools and related open practices as "best practices." This is owing to our own experiences and studies demonstrating open research is "associated with increases in citations, media attention, potential collaborators, job opportunities, and funding opportunities" (McKiernan et al., 2016, p. 1). However, we are aware that this is a narrow definition of "best" and may benefit from critiques and revisions, including adjusting our focus to "agreed-upon practices."

- Who gets to decide what a best practice is? When is it appropriate for a journal, funder, PI, or other decision-maker to impose practices or tools through use of power? What opportunities are there for those impacted by such decisions to raise issues or get support, particularly when compliance is an unfunded mandate? Resistance often arises for good reasons that should be taken into account when making change decisions. Mandates may underplay or ignore resistors' reasons and miss valuable opportunities for reflection on the impact of new practices.

- How do we strike a balance between "best" and "good enough" practices? Researchers are often time- and resource-constrained, so asking for major changes to everyday practice may augment resistive behaviors. We might be best served by making requests that meet researchers in the middle and providing avenues for incremental changes.

\section{Acknowledgments}

This work was supported by the Gordon \& Betty Moore Foundation (GBMF3834) and Alfred P. Sloan Foundation (2013-10-27), as part of the Moore-Sloan Data Science Environments grant to the University of California, Berkeley. 


\section{References}

Folger R, Konovsky MA. Effects of procedural and distributive justice on reactions to pay raise decisions. Academy of Management. 1989; 32(1):115-130. https://www.jstor.org/ stable/256422.

Geiger RS, DeMasi O, Culich A, Zoglauer A, Das D, de la Guardia FH, Ottoboni K, Fenner M, Varoquaux N, Barter R, Barnes R, Stoudt S, Dorton S, van der Walt S. Best Practices for Fostering Diversity and Inclusion in Data Science. In: BIDS Best Practices in Data Science Series Berkeley Institute for Data Science; 2018.https://doi.org/10.31235/osf.io/8gsjz.

Geiger RS, Sholler D, Culich A, Martinez C, de la Guardia FH, Lanusse F, Ottoboni K, Stuart M, Vareth M, Varoquaux N, Stoudt S, van der Walt S. Challenges of Doing Data-Intensive Research in Teams, Labs, and Groups. In: BIDS Best Practices in Data Science Series Berkeley Institute for Data Science; 2018.https://doi.org/10.31235/osf.io/a7b3m.

Goodman A, Pepe A, Blocker AW, Borgman CL, Cranmer K, Crosas M, Di Stefano R, Gil Y, Groth P, Hedstrom M, Hogg DW, Kashyap V, Mahabal A, Siemiginowska A, Slavkovic A. Ten Simple Rules for the Care and Feeding of Scientific Data. PLoS Computational Biology. 2014 Apr; 10(4):e1003542. doi: 10.1371/journal.pcbi.1003542.

Joshi K. A model of users' perspective on change: the case of information systems technology implementation. MIS Quarterly. 1991; p. 229-242. https://www.jstor.org/stable/ 249384.

Lapointe L, Rivard S. A multilevel model of resistance to information technology implementation. MIS Quarterly. 2005; 29(3). https://www.jstor.org/stable/25148692.

Marakas GM, Hornik S. Passive resistance misuse: overt support and covert recalcitrance in IS implementation. European Journal of Information Systems. 1996; 5(3):208-219. https://doi.org/10.1057/ejis.1996.26.

Markus ML. Power, politics, and MIS implementation. Communications of the ACM. 1983; 26(6):430-444. https://doi.org/10.1145/358141.358148.

McFarlin DB, Sweeney PD. Distributive and procedural justice as predictors of satisfaction with personal and organizational outcomes. Academy of Management. 1992; 35(3):626637. https://doi.org/10.5465/256489.

McKiernan EC, Bourne PE, Brown CT, Buck S, Kenall A, Lin J, McDougall D, Nosek BA, Ram K, Soderberg $\mathrm{CK}$, et al. Point of view: How open science helps researchers succeed. Elife. 2016; 5:e16800. https://doi.org/10.7554/eLife.16800.

Sandve GK, Nekrutenko A, Taylor J, Hovig E. Ten Simple Rules for Reproducible Computational Research. PLoS Computational Biology. 2013; 9(10). doi: 10.1371/journal.pcbi.1003285.

Sholler D, Das D, de la Guardia FH, Hoffmann C, Lanusse F, Garcia R, Geiger RS, McDevitt S, Peterson S, Stoudt S. Best Practices for Managing Turnover in Data Science Grouups, 
Teams, and Labs. In: BIDS Best Practices in Data Science Series Berkeley Institute for Data Science; 2019.https://doi.org/10.31235/osf.io/TBD.

Wilson G, Aruliah DA, Brown CT, Chue Hong NP, Davis M, Guy RT, Haddock SHD, Huff KD, Mitchell IM, Plumbley MD, Waugh B, White EP, Wilson P. Best Practices for Scientific Computing. PLoS Biology. 2014 Jan; 12(1):e1001745. doi: 10.1371/journal.pbio.1001745.

Wilson G, Bryan J, Cranston K, Kitzes J, Nederbragt L, Teal TK. Good enough practices in scientific computing. PLOS Computational Biology. 2017 Jun; 13(6):e1005510. doi: 10.1371/journal.pcbi.1005510. 